\title{
The relationship between immature platelet fraction and severity of acute bronchiolitis
}

\author{
Murat Doğan ${ }^{1 \oplus}$, Mehmet Köse ${ }^{2 \oplus}$, Mehmet Adnan Öztürk ${ }^{1 \oplus}$, Melih Hangül ${ }^{1 \oplus}$, \\ Hümeyra Aslaner ${ }^{3 \odot}$ \\ Departments of ${ }^{1}$ Pediatric Emergency, ${ }^{2}$ Pediatric Pulmonology and ${ }^{3}$ Family Medicine, Erciyes University Faculty of Medicine, Kayseri, \\ Turkey.
}

\begin{abstract}
Background and objectives. Acute bronchiolitis is one of the most common reasons for hospitalization in infants. Although patients with acute bronchiolitis generally have a good prognosis, death can also occur. In this study, we evaluate the immature platelet fraction (IPF) as an indicator of the severity of acute bronchiolitis.

Methods. In our study, 179 patients diagnosed with acute bronchiolitis were divided into three groups as mild (n: $48 ; 26.8 \%)$, moderate (n: $104 ; 58.10 \%$ ) and severe (n: $27 ; 15.1 \%$ ) bronchiolitis. There were 80 healthy children in the control group. The diagnostic capacity of IPF and hematological parameters (platelet distribution width (PDW), mean platelet volume (MPV), white blood cell count (WBC), and platelet count (PLT)) values to predict severity of acute bronchiolitis was evaluated using receiver operating characteristic (ROC) curves and their respective areas under the curves (AUCs) calculated with 95\% confidence intervals.
\end{abstract}

Results. The IPF value of patients with acute bronchiolitis was significantly higher than the healthy group ( $\mathrm{p}$ $<0.001$ ). In addition, a positive correlation was observed between clinical severity of bronchiolitis and IPF. The ROC curve analysis indicated that the IPF cut-off point for predicting severity of acute bronchiolitis was $>3.2 \%$ (Sensitivity of $84 \%$, specificity of $97 \%$ ). We found that the AUCs for IPF, MPV, PDW, WBC and PLT were statistically significant for bronchiolitis relative to the healthy control group. The parameter with the greatest AUC value was IPF.

Conclusion. The IPF may present for diagnosing and evaluating the clinical severity of acute bronchiolitis in children.

Key words: acute bronchiolitis, immature platelet fraction, children.

Acute bronchiolitis is the most common lower respiratory tract infection in children younger than 1 year. ${ }^{1}$ Viruses are the most common cause of acute bronchiolitis, especially respiratory syncytial virus. Bronchiolitis is characterized by airway obstruction and edema, increased mucus production, and the loss of airway epithelial cells. The clinical severity of bronchiolitis ranges from mild cases that can be treated on an outpatient basis to severe cases that require mechanical ventilation or extracorporeal

\footnotetext{
Murat Doğan

doktormurat033@hotmail.com
}

Received 10th February 2021, revised 4th May 2021, accepted 13th May 2021. membrane oxygenation in intensive care units. ${ }^{2}$ Acute bronchiolitis might be associated with morbidity and mortality in children. Currently, there is no biomarker that can predict the severity of acute bronchiolitis. The lack of any single biomarker for acute bronchiolitis that may be applied across all clinical scenarios is a recurring problem and has led many clinicians and researchers to pursue alternative diagnostic strategies. $^{2}$

Platelets play an important role in hemostasis, inflammation, allergic reactions, and angiogenesis, as well as the repair and renewal of tissues. They also secrete mediators such as chemokines, cytokines, and coagulation factors, 
which provoke a strong inflammatory response and tissue regeneration. Platelet production in bone marrow increases and platelets are redistributed during the inflammatory response. ${ }^{3}$ In addition to platelet count (PLT), platelet indexes include platecrite, mean platelet volume (MPV), platelet distribution width (PDW), and a new inflammatory parameter called the immature platelet fraction (IPF). The IPF is the percentage of reticulated platelets that can be measured in the blood, and it may be used to quantify the production of bone marrow platelets. The IPF correlates directly with the thrombopoietic rate, increasing when platelet production rises and decreasing when production falls. Inflammation causes changes in the bone marrow, leading to increases in platelet production and specifically of the circulation of immature platelets. ${ }^{4-6}$ There may be a relationship between the number of immature platelet fraction (IPF) in circulation and the severity of inflammation. Diagnostic accuracy studies performed over the last few years suggest that IPF levels can provide clinically relevant information regarding inflammatory activity and disease prognoses. ${ }^{5-8}$ Here, we evaluate the relationship between changes in platelet parameters such as IPF and the clinical severity of acute bronchiolitis.

\section{Material and Methods}

A single center prospective case control study of children aged 1-24 months who were hospitalized with acute bronchiolitis was performed between December 2018 and May 2019. In accordance with American Academy of Pediatrics guidelines, a diagnosis of acute bronchiolitis was based on at least two of the following signs: chest retractions, tachypnea, and the first episode of wheezing or rales on auscultation following a viral upper respiratory tract infection in children aged younger than 24 month. ${ }^{9}$ During the study period, 330 acute bronchiolitis patients were admitted and 179 patients were included in the study. 80 children seen in Erciyes University Medical
School Children's Hospital in Social Pediatrics outpatient clinic for routine control were included in the study as the control group. The patients had no health problems at this time were taken as a control group, which were similar to the patient group in terms of age and gender. Before the study, the all groups were informed about the study and informed consent were taken. On admission, the clinical severity score (CSS) for acute bronchiolitis (i.e., a composite clinical score including respiratory rate, retraction, wheezing, and general condition) was used to evaluate patients, as previously described by Wang et al. ${ }^{10}$ An adapted score from Wang et al. used for disease severity. ${ }^{11}$ Moderate bronchiolitis was defined by a score of $>5$ and severe by a score of $>10$. Bronchiolitis severity score (BSS) was recorded for each patient at the time of presentation. Each patient with bronchiolitis was classified into one of three groups, depending on whether they had mild, moderate, or severe bronchiolitis. Inclusion criteria were: aged 1-24 months, first wheezing episode, no previous disease history, and no previous medication. Exclusion criteria were: chronic disease, premature birth, birth weight $<2500 \mathrm{~g}$, malnutrition, passive smoking, proven immune deficiency, proven or suspected acute bacterial infection, previous treatment with bronchodilators or corticosteroids, or having symptoms for more than 7 days.

\section{Data collection}

Complete blood count measurements (including white blood cells, MPV, and platelets) were recorded from the blood samples taken on the first day of hospitalization using a BC6800 analyzer (Mindray, Shenzhen, China). Eosinophil counts (\%) determined by automated blood analyzer. The IPF was recorded using flow cytometry and the reticulocyte/platelet channel of an automated hematology analyzer (Sysmex, Kobe, Japan) with a fluorescent dye containing polymethrin and oxazine. In cases of our study, blood samples were taken before starting steroid treatment. The IPF is the fraction (\%) of immature platelets in the total 
platelet population and reference range for the IPF has been determined $1-5 \%$. The range of IPF reference values in a healthy population is $1.1-6.1 \%$ (with Sysmex analyzer). ${ }^{12,13}$ Data from each patient recorded in the emergency room included: age, sex, disease history, medication, birth history, whether this was the first attack of bronchiolitis, weight, vital signs (i.e., heart rate, respiratory rate, tympanic temperature, and oxygen saturation when breathing ambient air, which was measured using pulse oximetry and expressed as $\mathrm{SpO}_{2}$ ). Complete blood counts and IPF data were obtained from blood samples taken for routine testing of these children at the time of admission. This study was conducted with the approval of the Ethical Committee of Erciyes University Faculty of Medicine on 23/02/2018 (Project number: 2018/91). All study procedures were performed in accordance with the ethical principles of the 1964 Declaration of Helsinki. No financial support has been received from any institution or organization.

\section{Statistical methods}

In our study, power analysis was performed using $G$ * Power 3.1 analysis program to determine the samplesize. The number of patient to participate in the study with a population size of 330 was determined as a minimum of 178. ( $\alpha$-value: $0.050, \beta$-value: 0.800$)$. Statistical analyses were performed using SPSS software (ver. 21.0; IBM, Chicago, IL, USA). Continuous variables are expressed as medians (minimummaximum) and categorical variables as values and percentages. Categorical data were compared using chi-square tests. For nonnormally distributed data, the Mann-Whitney $\mathrm{U}$ test was used to determine whether or not differences between groups were statistically significant. Spearman's method was used to evaluate correlations between the IPF and other blood parameters if variables were not normally distributed. The most discriminating biomarkers for acute bronchiolitis were identified by drawing receiver operating characteristic (ROC) curves for each biomarker, and calculating sensitivity and specificity, as well as positive and negative predictive values. Areas under the curves (AUCs) for IPF, PLT, MPV, PDW, and white blood cell count (WBC) were evaluated for the acute bronchiolitis versus the control group. Logistic regression was used to identify associated factors and to calculate odds ratios and $95 \%$ confidence intervals. A $p$-value $<0.05$ was considered statistically significant.

\section{Results}

In total, 179 patients aged 1-24 months were included in this study, with a median age of 8 months (range, 1-24 months): 116 (64.800\%) of these patients were male and $63(35.200 \%)$ were female. The control group included 80 healthy children, also with a median age of 8 months (range, 2-24 months): 49 (61.200\%) of them were male and $31(38.800 \%)$ were female. The patient and control group did not differ significantly in age or sex $(p=0.619$ and $p=$ 0.583 , respectively). Patients had significantly higher WBC, PLT, MPV, IPF, and PDW values compared with the control group $(p=0.000)$. The frequency of eosinophilia did not differ significantly between the patient and control groups ( $p=0.756$; Table I). At admission, the median body temperature of patients was $37^{\circ} \mathrm{C}$ $\left(36-39.3^{\circ} \mathrm{C}\right)$ and their median oxygen saturation was 91\% (75-99\%). In total, 99 patients had a chest X-ray finding (e.g., diffuse interstitial consolidation or increased aeration), 68 patients were given antibiotics, and 71 patients received steroid therapy (all patients in the severe group and $42.300 \%$ of patients in the moderate group). All patients were provided with supportive therapy such as hydration, oxygen, and salbutamol nebules if indicated. The patient group included 48 (26.800\%), 104 (58.100\%), and $27(15.100 \%)$ children classified as having mild, moderate, and severe bronchiolitis, respectively. The median CSS was 6 (range, 2-12). No significant correlation was found between acute bronchiolitis CSS and age, sex, body temperature, oxygen saturation (\%), PLT, $\mathrm{WBC}$, proportion of lymphocytes, or proportion of eosinophils $(p>0.05)$. The MPV value of the 
Table I. Comparison of demographic and laboratory characteristics of the patients and healty group.

\begin{tabular}{lccc}
\hline & $\begin{array}{c}\text { Patient Group Median } \\
\text { (Min-Max) }\end{array}$ & $\begin{array}{c}\text { Healty Group Median } \\
(\text { Min-Max })\end{array}$ & $\mathrm{p}$ \\
\hline Age $($ month) & $8(1-24)$ & $8(2-24)$ & 0.619 \\
Sex male $(\mathrm{n})(\%)$ & $116(64.800)$ & $49(61.200)$ & 0.583 \\
IPF $(\%)$ & $5.500(0.700-18.800)$ & $1.950(0.800-4.100)$ & $<0.001$ \\
MPV (fL) & $9.400(8-11.800)$ & $8.600(7.400-9.900)$ & $<0.001$ \\
PDW $(\%)$ & $9.800(7.700-15.400)$ & $9.250(7.700-12.100)$ & 0.027 \\
WBC $\left(10^{9} / \mathrm{L}\right)$ & $10.260(2.040-24.440)$ & $6.720(3.140-11.500)$ & $<0.001$ \\
PLT $\left(10^{9} / \mathrm{L}\right)$ & $266(74-631)$ & $288(101-449)$ & $<0.001$ \\
Eozinofil $(\%)$ & $0.300(0.000-9.100)$ & $0.200(0.000-9.100)$ & 0.756 \\
\hline
\end{tabular}

Mann Whitney U test was used. $\mathrm{p}<0.05$ was considered statistically significant.

IPF: immature platelet fraction, MPV: mean platelet volume, PDW: platelet distribution width, WBC: white blood cell count, PLT: platelet.

Table II. Comparison of the clinical severity of acute bronchiolitis and laboratory findings.

\begin{tabular}{lcccc}
\hline & $\begin{array}{c}\text { Mild Median } \\
(\text { Min-Max })\end{array}$ & $\begin{array}{c}\text { Moderate Median } \\
(\text { Min-Max })\end{array}$ & $\begin{array}{c}\text { Severe Median } \\
(\text { Min-Max })\end{array}$ & $\mathrm{p}$ \\
\hline IPF $(\%)$ & $3.350(0.700-9.600)$ & $5.600(1.400-18.700)$ & $10.800(3.600-18.800)$ & $<0.001$ \\
MPV (fL) & $9(8.500-11.300)$ & $9.500(8.000-11.800)$ & $10.200(8.700-11.700)$ & $<0.001$ \\
PDW $(\%)$ & $8.90(7.800-14)$ & $9.950(7.700-13.600)$ & $11.100(8.400-15.400)$ & $<0.001$ \\
WBC $\left(10^{9} / \mathrm{L}\right)$ & $4.090(2.810-31.000)$ & $11.570(4-23.800)$ & $9.790(4.190-23.800)$ & 0.422 \\
PLT $\left(10^{\%} / \mathrm{L}\right)$ & $220.500(88-430)$ & $231(74-631)$ & $218.00(88-631)$ & 0.335 \\
Eozinofil $(\%)$ & $0.500(0.000-4.700)$ & $0.150(0.000-9.100)$ & $0.400(0.000-9.100)$ & 0.280 \\
\hline
\end{tabular}

Kruskal Wallis Test and Post Hoc Dunn correction were used. $\mathrm{p}<0.05$ was considered statistically significant.

IPF: immature platelet fraction, MPV: mean platelet volume, PDW: platelet distribution width, WBC: white blood cell count, PLT: platelet.

mild clinical severity group was significantly lower than those of the moderate $(p=0.002)$ and severe $(p=0.000)$ group, but MPV values did not differ significantly between the moderate and severe groups $(p=0.142)$. IPF values differed significantly among the mild, moderate, and severe groups $(p<0.001)$. A positive correlation was observed between the CSS and the IPF ( $p$ $<0.001)$. The mild clinical severity group had a significantly lower PDW compared with the moderate and severe groups $(p=0.000)$, but the PDW values of the moderate and severe groups did not differ significantly (Table II).

ROC curve analyses were used to evaluate the performance of each biomarker in distinguishing acute bronchiolitis patients from controls, and in assessing acute bronchiolitis severity. The AUC for IPF, MPV, PDW, WBC, and PLT was
$0.950,0.850,0.580,0.790$, and 0.710 , respectively. ROC curve analysis suggested that the cut-off for using IPF to predict bronchiolitis was $>3.2$, with a sensitivity of $84 \%$ and a specificity of $97 \%$. The positive and negative predictive values of the IPF were $98.700 \%$ and $73.500 \%$, respectively. The AUC was greatest for IPF (Fig. 1). IPF, MPV, and PDW ROC curves showed significant sensitivity and selectivity in patients $(p<0.001$; Table III). For all patients, this was the first bronchiolitis attack.

\section{Discussion}

We found that the IPF, which is a new inflammatory platelet index, was greater in patients with acute bronchiolitis than in healthy individuals and was positively correlated with the clinical severity of acute bronchiolitis. 
Table III. ROC analysis of inflammation markers in the prediction of acute bronchiolitis.

\begin{tabular}{lccccccc}
\hline Parameter & Cut-off & Sensitivity $\%$ & Specificity $\%$ & PPV & NPV & AUC $(95 \%$ CI) & p \\
\hline IPF $(\%)$ & $>3.200$ & 84 & 97 & 98.700 & 73.500 & $0.950(0.910-0.970)$ & $<0.001$ \\
MPV (fL) & $>9$ & 78 & 72 & 86.400 & 59.700 & $0.850(0.800-0.890)$ & $<0.001$ \\
PDW $(\%)$ & $>10.100$ & 44 & 75 & 79.800 & 37.500 & $0.58(0.520-0.640)$ & 0.017 \\
WBC $\left(10^{9} / \mathrm{L}\right)$ & $>9.940$ & 56 & 95 & 96.200 & 49.600 & $0.790(0.730-0.830)$ & $<0.001$ \\
PLT $\left(10^{\%} / \mathrm{L}\right)$ & $>424$ & 37 & 97 & 97.100 & 41.100 & $0.710(0.650-0.760)$ & $<0.001$ \\
\hline
\end{tabular}

IPF: immature platelet fraction, MPV: mean platelet volume, PDW: platelet distribution width, WBC: white blood cell count, PLT: platelet, PPV: positive predictive value, NPV: negative predictive value, AUC (95\% CI): area under the receiver operating characteristic curve ( $95 \%$ confidence interval).

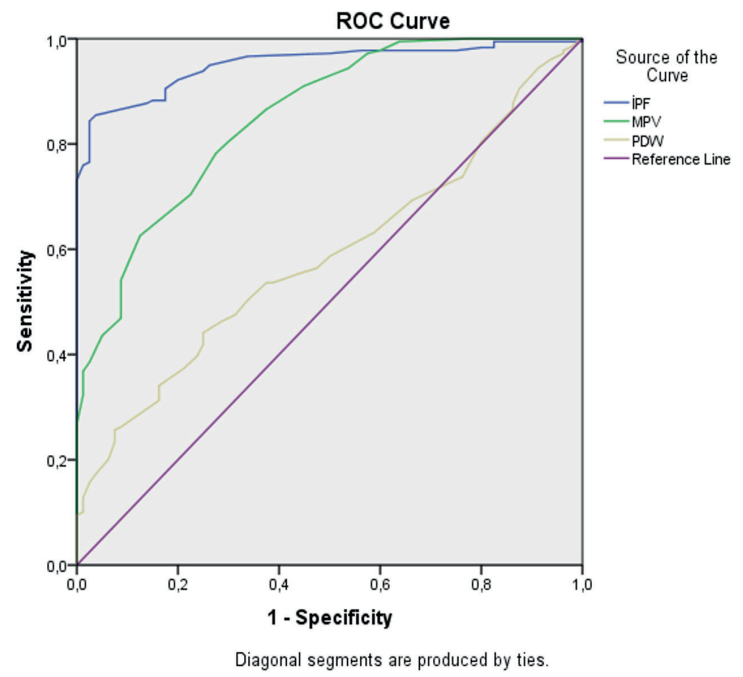

Fig. 1. Receiver operating characteristic curve (ROC) analysis of Immature platelet fraction (IPF), Mean platelet volume (MPV), Platelet distribution width (PDW).

To our knowledge, this is the first study to investigate the relationship between acute bronchiolitis and IPF. Some cytokines and mediators are activated as part of the response to inflammatory conditions. Therefore, we think that there may be a positive correlation between the severity of inflammation and the immature platelet fraction in the bone marrow. One of the biochemical and hematological systemic inflammatory markers is supposed to be IPF, an index of platelet activation and production rate in the bone marrow. ${ }^{3}$ Diagnostic accuracy studies performed over the last few years suggest that IPF levels can provide clinically relevant information regarding inflammatory activity and disease prognoses. ${ }^{7}$ De Blasi et $a .^{6}$ reported that IPF levels increased in patients with sepsis before sepsis was observed clinically, and they also found that IPF was a better biomarker for sepsis than procalcitonin or C-reactive protein. Park et al. ${ }^{5}$ found that IPF levels were significantly higher in patients with sepsis than in individuals who did not have sepsis. Rodolfo et al. ${ }^{7}$ evaluated the IPF as a biomarker for sepsis diagnosis and severity and found that it was correlated with sepsis severity scores and had the highest diagnostic accuracy for sepsis among all the clinical and laboratory parameters assessed. Another study found that IPF was strongly positively correlated with MPV and PDW, and attributed this to an increase in the number of larger and wider platelets that appeared after the destruction of pro-inflammatory cytokines and endotoxins as part of a severe inflammatory response. ${ }^{8}$ Together, these findings suggest that IPF levels increase in inflammatory diseases such as sepsis and may be used as an indicator of the inflammatory response. In our study, there were significant differences in inflammatory markers (IPF, MPV, PLT, and WBC) between acute bronchiolitis patients and healthy individuals. These inflammation markers can be used to predict the clinical severity of acute bronchiolitis, but the marker with the highest sensitivity and specificity was IPF (AUC:0.950). Our observation that the IPF was greater in patients with acute bronchiolitis than in healthy individuals is consistent with previous studies. The clinical severity of acute bronchiolitis was positively correlated with the IPF. Therefore, patients with acute bronchiolitis and high IPF values (>3.2) should be monitored closely after admission to the emergency department. 
Platelets play an important role in inflammatory responses, and the PLT increases in many inflammatory diseases. Studies have found that patients with acute bronchiolitis have higher PLTs than healthy individuals. ${ }^{14,15}$ Our patient group had significantly greater PLT values than our healthy control group, and this observation is consistent with previous studies. Our severe bronchiolitis group had higher PLT values compared to our mild bronchiolitis group, but the difference was not statistically significant. During an inflammatory response, depolymerization occurs in the microtubular structure of platelets, and changes also occur in the structure of actin that becomes polymerized; consequently, platelets change their shapes and these changes are reflected in the MPV. ${ }^{15}$ Changes in MPV can be observed before changes in platelet number, so changes in MPV may be useful for predicting inflammation at an early stage and determining its subsequent severity. ${ }^{16-18}$ Among our study population, the MPV was significantly higher in those with moderate and severe bronchiolitis than in those with mild bronchiolitis or in healthy controls, so MPV values may be used to estimate prognoses and the severity of acute bronchiolitis; patients with an MPV value greater than $9 \mathrm{fL}$ should be monitored carefully for clinical deterioration. Several previous studies have evaluated the relationship between acute bronchiolitis and MPV. Gökçe et al. ${ }^{3}$ found that patients with acute bronchiolitis had higher MPVs than their healthy counterparts, but found no statistically significant differences in MPVs between different bronchiolitis groups. Higher MPVs were observed in children with influenza A respiratory tract infections than in healthy children. ${ }^{19}$ MPVs were also found to be higher in children with pneumonia, asthma, or sepsis than in healthy children. ${ }^{20-22}$ Gasparyan et al. ${ }^{23}$ found that MPV values were increased in patients with mild inflammation, and they attributed this finding to the transfer of large activated platelets to the site of infection as the severity of inflammation increased. Together, these results suggest that the MPV may also be used to estimate prognoses and the severity of other inflammatory diseases.

PDW, another platelet volume index, shows the variation in platelet diameters and varies by platelet activation. An increase in PDW may occur as a result of swelling, disruption, or platelet immaturity. ${ }^{15}$ In our study, PDW values were significantly greater in patients with moderate and severe bronchiolitis cases than in those with mild bronchiolitis or healthy controls. Ergül et al. $^{15}$ found greater PDW values in children with acute bronchiolitis than in healthy children. PDW may also be greater in patients with sepsis than in healthy individuals, and may be used as a prognostic indicator for patients with sepsis. ${ }^{22-24}$

There are several limitations to the current study. Firstly, our study was not a multi-center study. In addition, the study population was not evaluated for the type of virus and the patients did not have other inflammatory markers such as CRP and procalcitonin.

In conclusion, the IPF is a novel indicator of inflammation, and it is also a new marker for evaluating the clinical severity of acute bronchiolitis. Larger prospective studies are needed to clarify the clinical significance of using IPF values to assess patients with acute bronchiolitis.

\section{Ethical approval}

This study was conducted with the approval of the Ethical Committee of Erciyes University Faculty of Medicine on 23/02/2018 (Project number: 2018/91).

\section{Author contribution}

The authors confirm contribution to the paper as follows: study conception and design: MD, MAÖ, MK; data collection: MD, MH, MK; analysis and interpretation of results: $\mathrm{HA}, \mathrm{MD}$, MAÖ, MK; draft manuscript preparation: MD, MAÖ, MK, MH, HA. All authors reviewed the results and approved the final version of the manuscript. 


\section{Source of funding}

None.

\section{Conflict of interest}

The author declares that there are no conflict of interests.

\section{REFERENCES}

1. Fleming PF, Richard S, Waterman K, et al. Medical retrieval and needs of infants with bronchiolitis: an analysis by gestational age. J Paediatr Child Health 2013; 49: 227-231. https://doi.org/10.1111/jpc.12025

2. Brown PM, Schneeberger DL, Piedimonte G. Biomarkers of respiratory syncytial virus (RSV) infection: specific neutrophil and cytokine levels provide increased accuracy in predicting disease severity. Paediatr Respir Rev. 2015; 16: 232-240. https://doi.org/10.1016/j.prrv.2015.05.005

3. Gökçe Ş, Kurugöl Z, Suner A. The role of mean platelet volume in the early detection o f acute bronchiolitis: a prospective study. Clin Respir J 2018; 12: 2513-2518. https://doi.org/10.1111/crj.12947

4. Ault KA, Rinder HM, Mitchell J, Carmody MB, Vary CP, Hillman RS. The significance of platelets with increased RNA content (reticulated platelets): a measure of the rate of thrombopoiesis. Am J Clin Pathol 1992; 98: 637-646. https://doi.org/10.1093/ ajcp/98.6.637

5. Park SH, Ha SO, Cho YU, Park CJ, Jang S, Hong SB. Immature platelet fraction in septic patients: clinical relevance of immature platelet fraction is limited to the sensitive and accurate discriminationof septic patients from non-septic patients, not to the discrimination of sepsis severity. Ann Lab Med 2016; 36: 1-8. https://doi.org/10.3343/alm.2016.36.1.1

6. De Blasi RA, Cardelli P, Costante A, Sandri M, Mercieri M, Arcioni R. Immature platelet fraction in predicting sepsis in critically ill patients. Intensive Care Med 2013; 39: 636-643. https://doi.org/10.1007/ s00134-012-2725-7

7. Enz Hubert RM, Rodrigues MV, Andreguetto BD, et al. Association of the immature platelet fraction with sepsis diagnosis and severity. Sci Rep 2015; 5: 8019. https://doi.org/10.1038/srep08019

8. Djuang MH, Ginting F, Hariman H. Immature platelet fraction in bacterial sepsis severity assessment. IOP Conf. Series: Earth and Environmental Science 2018; 125: 012024. https://doi.org/10.1088/1755$1315 / 125 / 1 / 012024$
9. American Academy of Pediatrics Subcommittee on Diagnosis and Management of Bronchiolitis. Diagnosis and management of bronchiolitis. Pediatrics 2006; 118: 1774-1793. https://doi. org/10.1542/peds.2006-2223

10. Wang EE, Milner RA, Navas L, Maj H. Observer agreement for respiratory signs and oximetry in infants hospitalized with lower respiratory infections. Am Rev Resp Dis 1992; 145: 106-115. https://doi.org/10.1164/ajrccm/145.1.106

11. Bressan S, Balzani M, Krauss B, Pettenazzo A Zanconato S, Baraldi E. High-flow nasal cannula oxygen for bronchiolitis in a pediatric ward: a pilot study. Eur J Pediatr 2013; 172: 1649-1656. https://doi. org/10.1007/s00431-013-2094-4

12. Briggs C, Kunka S, Hart D, Oguni S, Machin SJ. Assessment of an immature platelet fraction IPF in peripheral thrombocytopenia. Br J Haematol 2004; 126: 93-99. https://doi.org/10.1111/j.13652141.2004.04987.x

13. Hoffmann JJ. Reticulated platelets: analytical aspects and clinical utility. Clin Chem Lab Med 2014; 52: 1107-1117. https://doi.org/10.1515/cclm-2014-0165

14. Yeaman MR. Platelets: at the nexus of antimicrobial defence. Nat Rev Microbiol 2014; 12: 426-437. https:// doi.org/10.1038/nrmicro3269

15. Ergül AB, Torun YA, Uytun S, Aslaner H, Kisaaslan AP, Şerbetçi MC. Reduction in mean platelet volume in children with acute bronchiolitis. Turk Pediatri Ars 2016; 51: 40-45. https://doi.org/10.5152/ TurkPediatriArs.2016.3140

16. Andrews RK, Berndt MC. Platelet physiology and thrombosis. Thromb Res 2004; 114: 447-453. https:// doi.org/10.1016/j.thromres.2004.07.020

17. Bessman JD, Gilmer PR, Gardner FH. Use of mean platelet volume improves detection of platelet disorders. Blood Cells 1985; 11: 127-135.

18. Kisacik B, Tufan A, Kalyoncu U, et al. Mean platelet volume (MPV) as an inflammatory marker in ankylosing spondylitis and rheumatoid arthritis. Joint Bone Spine 2008; 75: 291-294. https://doi. org/10.1016/j.jbspin.2007.06.016

19. Fei Y, Zhang H, Zhang C. The application of lymphocyte/platelet and mean platelet volume/ platelet ratio in influenza A infection in children. J Clin Lab Anal 2019; 33: e22995. https://doi. org/10.1002/jcla.22995

20. Karadag-Oncel E, Ozsurekci Y, Kara A, Karahan S, Cengiz AB, Ceyhan $M$. The value of mean platelet volume in the determination of community acquired pneumonia in children. Ital J Pediatr 2013; 39: 16. https://doi.org/10.1186/1824-7288-39-16 
21. Dogru M, Aktas A, Ozturkmen S. Mean platelet volume increased in children with asthma. Pediatr Allergy Immunol 2015; 26: 823-826. https://doi. org/10.1111/pai.12381

22. Guclu E, Durmaz Y, Karabay O. Effect of severe sepsis on platelet count and their indices. Afr Health Sci 2013; 13: 333-338. https://doi.org/10.4314/ahs. v13i2.19
23. Gasparyan AY, Ayvazyan L, Mikhailidis DP, Kitas GD. Mean platelet volume: a link between thrombosis and inflammation? Curr Pharm Des 2011; 17: 47-58. https://doi.org/10.2174/138161211795049804

24. Akarsu S, Taskin E, Kilic M, et al. The effects of different infectious organisms on platelet counts and platelet indices in neonates with sepsis: is there an organism-specific response? J Trop Pediatr 2005; 51: 388-391. https://doi.org/10.1093/tropej/fmi031 